EPJ Web of Conferences 52, 01003 (2013)

DOI: $10.1051 /$ epjconf/20135201003

(C) Owned by the authors, published by EDP Sciences, 2013

\title{
Characteristics of hadronic interactions in the forward direction
}

\author{
I. Katkov ${ }^{1,2, a}$ \\ ${ }^{1}$ Institut für Experimentelle Kernphysik, Karlsruhe Institut für Technologie (KIT), Karlsruhe, Germany \\ ${ }^{2}$ on behalf of the CMS collaboration
}

\begin{abstract}
A selection of results obtained with very forward detectors of the CMS experiment at LHC is presented. Studies of the energy flow, $\mathrm{d} E / \mathrm{d} \eta$, are extended into very forward region with CASTOR calorimeter $(-6.6<\eta<5.2)$ for pp collisions at 3 different center-of-mass energies $(0.9,2.76$ and $7 \mathrm{TeV})$ and $\mathrm{PbPb}$ collisions at $2.76 \mathrm{~A} \mathrm{TeV}$. Results are compared to hadronic interaction models as implemented in standard collider physics generators and generators used in cosmic-ray physics.
\end{abstract}

\section{Introduction}

At the collider energy frontier and with the most complete detector pseudorapidity coverage the CMS experiment is a perfect laboratory to uncover the long-awaited signatures of a possible new parton dynamics regime at low $x$. Benchmark measurements performed in CMS provide valuable information for standard collider Monte Carlo model tuning. In particular interesting is testing against the data the cosmic-ray hadronic Monte Carlo models (EPOS, QGSJET, SYBILL) which are assumed to provide best extrapolation up to highest interaction energies.

\section{CMS detector}

The central feature of the Compact Muon Solenoid (CMS) apparatus is a superconducting solenoid of $6 \mathrm{~m}$ internal diameter, providing a magnetic field of $3.8 \mathrm{~T}$. Within the field volume are an inner silicon pixel and strip tracker that measures charged particles in the range $|\eta|<2.5$, a lead tungstate crystal electromagnetic calorimeter, and a brass/scintillator hadron calorimeter. Muons are measured in gas-ionization detectors embedded in the steel return yoke. Extensive forward calorimetry complements the coverage provided by the barrel and endcap detectors. For more details on CMS see [1].

Cherenkov calorimetry was chosen in CMS for the forward detectors. The HF calorimeters cover $2.9<|\eta|<5.2$ using quartz fibers embedded within a steel absorber. The very forward angles are covered by the CASTOR $(-6.6<$ $\eta<-5.2)$ and Zero Degree $(|\eta|>8.1)$ calorimeters. The ZDC and CASTOR [2] calorimeters are made of quartz fibers/plates embedded in tungsten absorbers. The CASTOR has 16 azimuthal sectors and 14 longitudinal modules. Stacks of tungsten/quartz plates readout with photomultiplier tubes in two mechanical structures are placed

\footnotetext{
a e-mail: katkov@kit.edu; on leave from Moscow State University
}

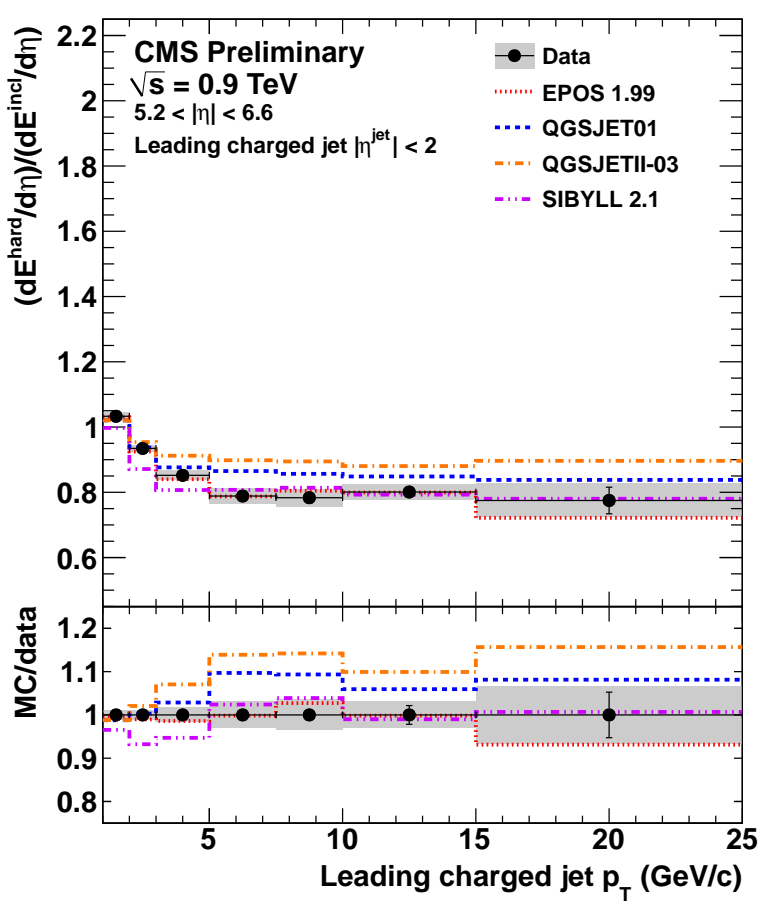

Figure 1: Hard-to-inclusive ratios measured in pp collisions at $900 \mathrm{GeV}$.

very close to the beam pipe at a distance of $14.4 \mathrm{~m}$ away from the nominal interaction point. The overall longitudinal depth of the calorimeter corresponds to 10 interaction lengths.

\section{Study of the underlying event at forward rapidity in pp collisions}

The basic quantities measured in the study are the ratios of energy pseudorapidity density in CASTOR calorime- 


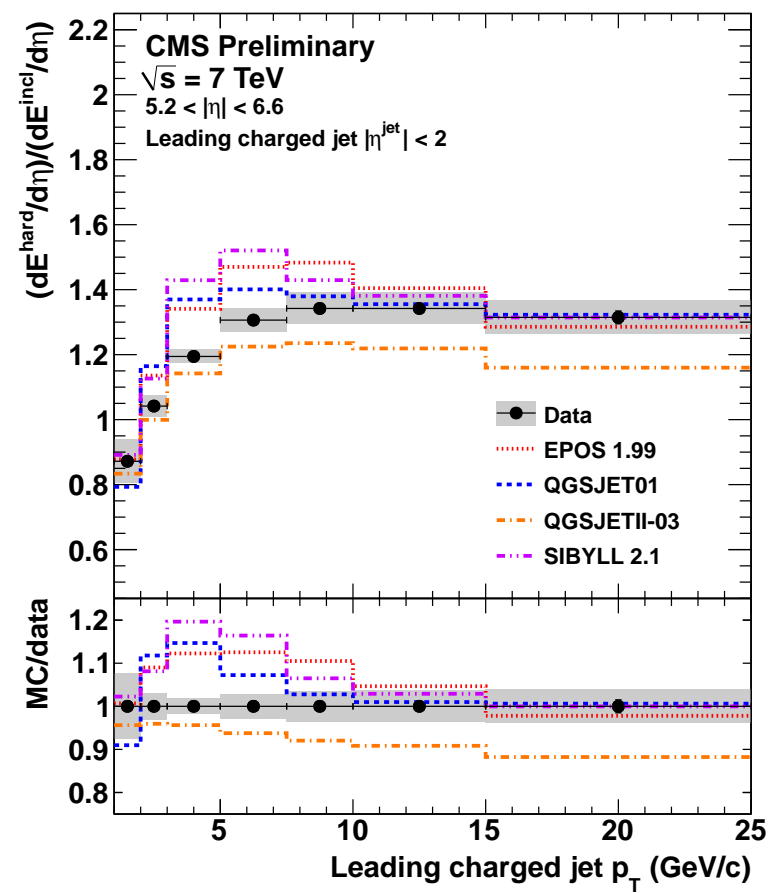

Figure 2: Hard-to-inclusive ratios measured in pp collisions at $7 \mathrm{TeV}$.

ter. Two classes of events are selected: non-diffractive minimum bias events and events with a hard scale where mid-rapidity jets (track jets) are reconstructed using anti$k_{\mathrm{T}}$ algorithm applied to tracks fitted to a primary vertex with transverse momentum of at least $0.3 \mathrm{GeV} / c$ [3]. The so called hard-to-inclusive ratios of energy are calculated using these two event classes. In ratios correlated systematic uncertainties, and in particular the absolute calibration uncertainty of CASTOR, cancel. Given the fact that CASTOR is placed close to the beam pipe, effects of deviation from non-ideal geometry need to be carefully estimated. A conservative estimate of corresponding systematic error gives the biggest contribution to the overall systematic uncertainty of the measurement. Hadron level corrections are performed. To reduce the hadron level correction factors, the hadron level is defined using cuts on $\xi_{\mathrm{X}}, \xi_{\mathrm{Y}}$ variables (scaled masses of hadronic systems on either side with respect to the largest rapidity gap in the event).

In Fig. 1-2 the hard-to-inclusive ratios are shown as a function of track jet $p_{\mathrm{T}}$. The results are shown for two different center-of-mass energies of $900 \mathrm{GeV}$ and $7 \mathrm{TeV}$. The data are compared to cosmic ray hadronic Monte Carlo models EPos, QGSJET01, QGSJETII, SYBILL. The data exhibit the typical underlying event behavior characterized by a rapid change of the energy pseudorapidity density at small track jet $p_{\mathrm{T}}$, followed by a plateau at larger $p_{\mathrm{T}}$. At $\sqrt{s}=7 \mathrm{TeV}$, the relative energy pseudorapidity density increases with $p_{\mathrm{T}}$, while at $\sqrt{s}=0.9 \mathrm{TeV}$ CASTOR is close to the beam fragmentation region and hence increased jet and underlying event activity at mid-rapidity implies depletion of the energy in CASTOR. The data is bracketed

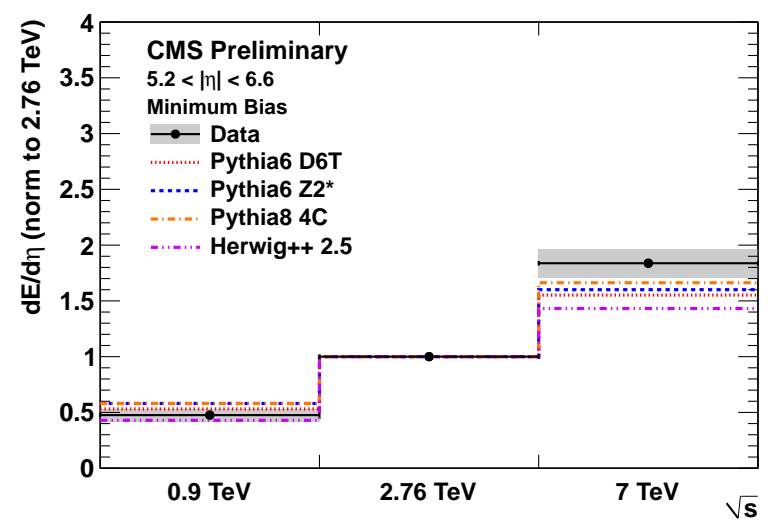

Figure 3: Normalized energy densities for 3 center-ofmass energies of pp collisions compared to PYTHIA tunes and HERWIG.

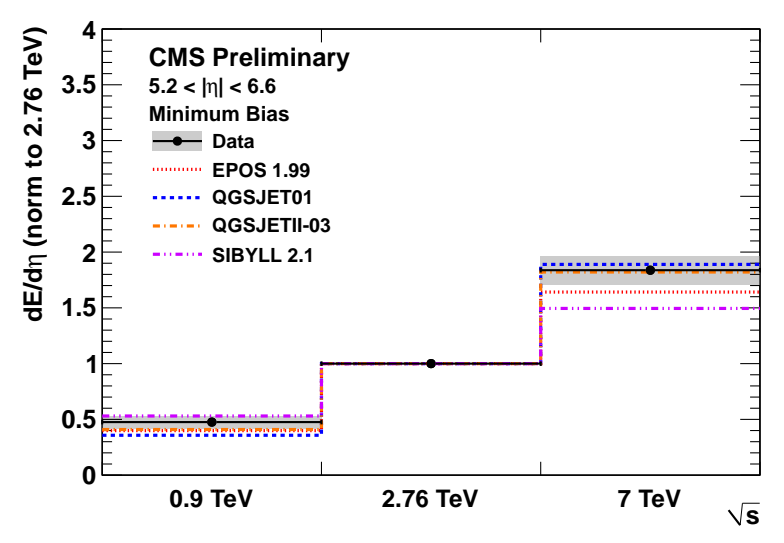

Figure 4: Normalized energy densities for 3 center-ofmass energies of pp collisions compared to cosmic-ray models.

by cosmic ray models of hadronic interactions that include no tuning to any LHC data.

Evolution of the energy pseudorapidity density measured in CASTOR with center-of-mass energy is shown for minimum bias events in Fig. 3-4. The data are normalized to the result at $2.76 \mathrm{TeV}$. In Fig. 3 the data are compared to HERWIG and PYTHIA Monte Carlo generator tunes. In Fig. 4 the comparison to cosmic ray models of hadronic interactions is shown. The cosmic ray models perform better in describing the energy evolution.

\section{Measurement of $\mathrm{d} E / \mathrm{d} \eta$ in $\mathbf{P b P b}$ collisions}

Using minimum bias $\mathrm{PbPb}$ collision events collected by CMS at nominal magnetic field at center-of-mass energy of $2.76 \mathrm{TeV}$ per nucleon it is possible to extend the absolute measurement of the energy flow, $\mathrm{d} E / \mathrm{d} \eta$, into the region close to the beam rapidity $\left(y_{\text {beam }}=7.99\right)[4,5]$. The CASTOR energy scale is set via a cross-calibration procedure according to energy pseudorapidity density measured 


\section{CMS PRELIMINARY}

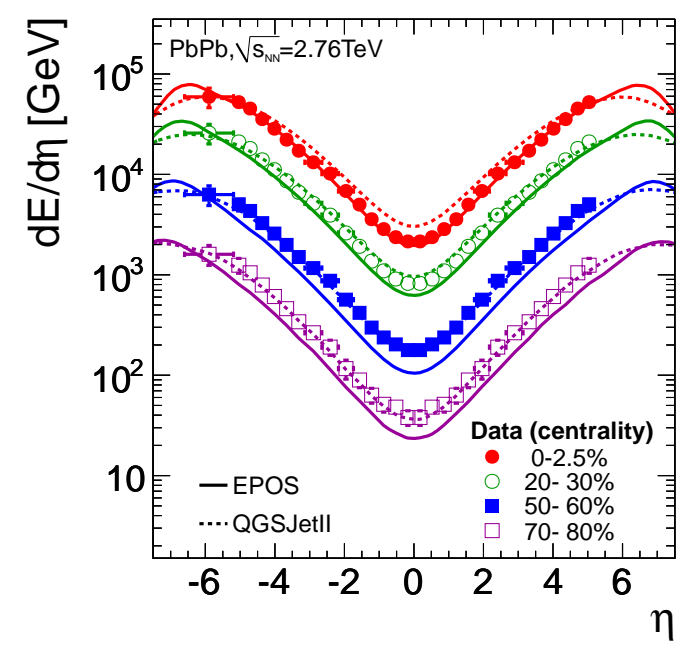

Figure 5: Corrected to hadron level energy pseudorapidity density, $\mathrm{d} E / \mathrm{d} \eta$, for different centralities of $\mathrm{PbPb}$ collisions at $2.76 \mathrm{TeV}$.

in the HF calorimeter using an independent data set of minimum bias pp collision events at $7 \mathrm{TeV}$ center-of-mass energy. The data are hadron level corrected, with typical values of correction factors being around 1.7 that vary only within a couple of percent with centrality. All stable particles with a lifetime $c \tau>10 \mathrm{~mm}$ define the hadron, or particle, level in the analysis. The overall systematic uncertainty on the energy scale is conservatively estimated to be $22 \%$ including, among the main contributions, the precision in determination of detector geometry and the uncertainties of the calibration procedure. The front part of the calorimeter, which comprises 3.2 interaction lengths, is used in the analysis to ensure moderate uncertainties of the calibration procedures. The calorimeter noise is low, less of a percent level of the signals observed in peripheral hadronic collision events.

In Fig. 5 the result of the energy flow measurement with complete CMS calorimetry including the forward calorimeters, HF and CASTOR, is shown. The $\mathrm{d} E / \mathrm{d} \eta$ is shown as a function of pseudorapidity for events selected from four different centrality classes. The data are symmetrized with respect to $\eta=0$ for all points except for the CASTOR one. A good description of both centrality and rapidity dependence is a challenge for the Monte Carlo models, including EPOS and QGSJETII.3 shown in Fig. 5. The EPOs generator used for the comparison is tuned to LHC pp collision data.

Several quantities can be derived from the measured energy pseudorapidity density to highlight particular aspects of the measurement. To calculate $R_{\mathrm{PC}}$ the energy (as a function of rapidity and centrality) is scaled by the corresponding number of participating nucleons, $N_{\text {part }}$, and then normalized to the result for the $2.5 \%$ most central events where $N_{\text {part }}^{\max }=394$. The shape of the $R_{\mathrm{PC}}$ centrality

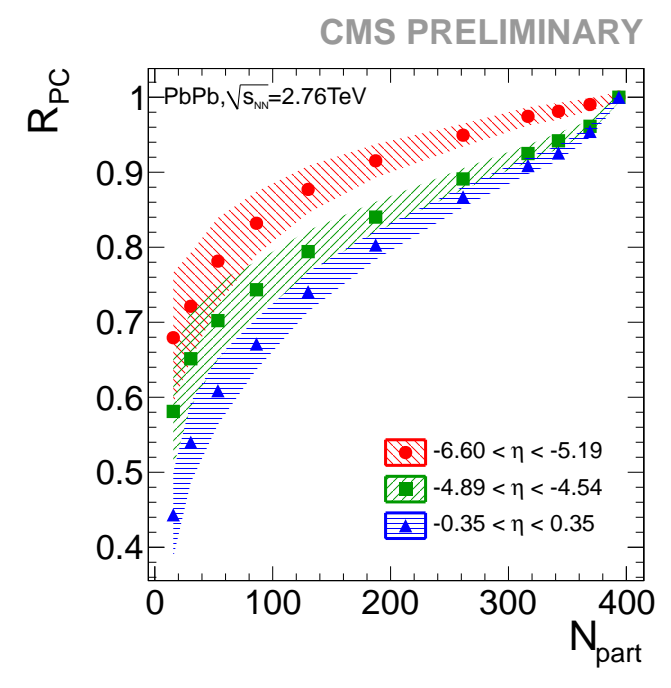

Figure 6: The normalized energy flow per participant, $R_{\mathrm{PC}}$, measured in $\mathrm{PbPb}$ collisons at $2.76 \mathrm{TeV}$.

dependence is expected to be sensitive to the geometry of the $\mathrm{PbPb}$ collisions. In $R_{\mathrm{PC}}$ measurement correlated uncertainties, and in particular energy scale, gracefully cancel. The dominant contribution to the systematic uncertainty of the $R_{\mathrm{PC}}$ is due to uncertainty on the $N_{\text {part }}$ determination. Flattening of the $R_{\mathrm{PC}}$ centrality dependence in the forward pseudorapidity bins can be seen in Fig. 6. It is worth noting here that at higher pseudorapidities lower $x$ values are probed.

The beam fragmentation region can be studied in detail by introducing $y^{\prime}=|\eta|-y_{\text {beam }}$ and limiting $y^{\prime}$ to the range $-y_{\text {beam }}<y^{\prime}<0$. The $\mathrm{d} E / \mathrm{d} y^{\prime}$ for the $10 \%$ most central events is shown in Fig. 7. The distribution in Fig. 7 represents the dispersion process of the $\mathrm{Pb}$ projectile after a collision, while before the collision the projectile can be represented as a $\delta$-function at beam rapidity. A reliable extrapolation (also indicated in Fig. 7) to unobserved phasespace is possible as the CMS data points extend to close proximity to the beam rapidity. Upon the extrapolation the average energy-weighted pseudorapidity (relative to the beam rapidity) can be determined as $\langle y\rangle_{\mathrm{E}} \sim \int y^{\prime} \mathrm{d} E / \mathrm{d} y^{\prime} \mathrm{d} y^{\prime}$ with a systematic uncertainty below $15 \%$ for $10 \%$ most central events in case of an extreme assumption about the unobserved energy. The $\langle y\rangle_{\mathrm{E}}$ characterizes the energy balance in the fragmentation region. This observable can be used for model discrimination and confronted with results on baryon stopping obtained at RHIC.

\section{Summary}

First results obtained with CMS forward CASTOR calorimeter using $\mathrm{pp}$ and $\mathrm{PbPb}$ collision data are presented. The results explore the full potential of the CMS calorimetry coverage at the highest $\mathrm{pp}$ and $\mathrm{PbPb}$ collision energies. 


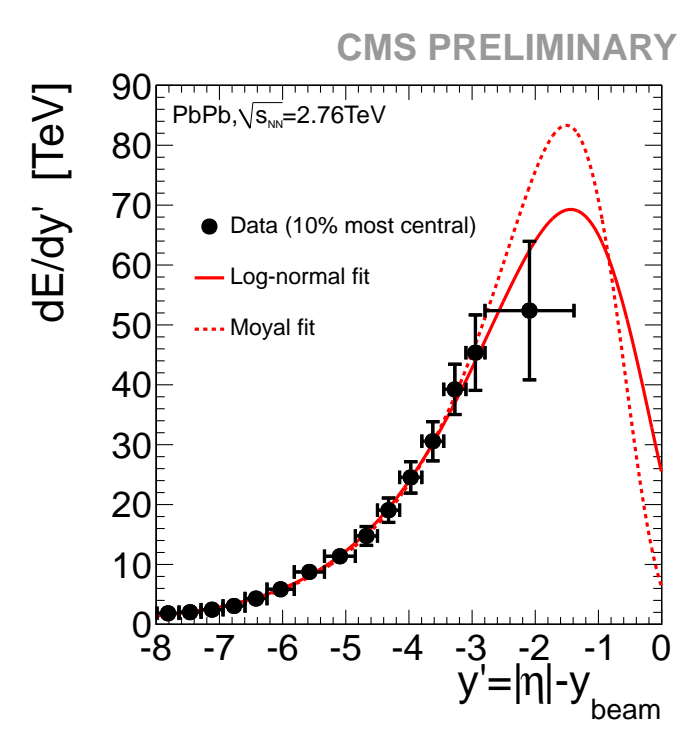

Figure 7: The energy pseudorapidity density, $\mathrm{d} E / \mathrm{d} y^{\prime}$, as a function of $y^{\prime}=|\eta|-y_{\text {beam }}$ for $10 \%$ most central $\mathrm{PbPb}$ collisions at $2.76 \mathrm{TeV}$.

Studies of energy flow ratios in pp collisions as a function of center-of-mass energy and hard scale complement measurements of underlying event done at central rapidities and provide valuable information for model tuning. Studies of the energy flow density, $\mathrm{d} E / \mathrm{d} \eta$, in $\mathrm{PbPb}$ collisions as a function of pseudorapidity and centrality provide new opportunities to investigate beam fragmentation region. These results are comparable to the most forward measurements at RHIC. Results presented in this article as well as the results to be obtained in the $\mathrm{pPb}$ collisions, planned to occur at LHC in January-February of 2013, are important reference points for the cosmic ray community.

\section{Acknowledgments}

Efforts of physicists, engineers and technicians, contributed to the construction, operation and support of the LHC and the CMS experiment, are gratefully acknowledged. This work was supported by the HelmholtzNachwuchsgruppen grant VH-NG-733 and partially supported by RF Presidential grant N 3920.2012.2 for the Leading Scientific Schools.

\section{References}

[1] S. Chatrchyan et al. [CMS Collaboration], The CMS experiment at the CERN LHC, JINST 3, S08004 (2008)

[2] V. Andreev et al., Performance Studies Of A FullLength Prototype For The Castor Forward Calorimeter At The Cms Experiment, Eur. Phys. J. C 67, 601 (2010)

[3] S. Chatrchyan et al. [CMS Collaboration], Study of the Underlying Event at Forward Rapidity in ProtonProton Collisions at the LHC, CMS-PAS-FWD-11-003 (2012)

[4] S. Chatrchyan et al. [CMS Collaboration], Measurement of the pseudorapidity and centrality dependence of the transverse energy density in $\mathrm{PbPb}$ collisions at $\sqrt{s_{N N}}=2.76 \mathrm{TeV}$, Phys. Rev. Lett. 109, 152303 (2012)

[5] S. Chatrchyan et al. [CMS Collaboration], Measurement of the pseudorapidity and centrality dependence of $\mathrm{d} E / \mathrm{d} \eta$ in $\mathrm{PbPb}$ collisions at $\sqrt{s_{N N}}=2.76 \mathrm{TeV}$ extending into the very forward phase-space, CMS-PASHIN-12-006 (2012) 